\title{
Impact of Climate Resilient Technologies on Socio-Economic Status of Farmers
}

\author{
Kamal Narayan", Narayan Sahu, Premlal Sahu and Kedar Nath Yadaw
}

Krishi Vigyan Kendra, Dantewada (C.G.) 494441, India

*Corresponding author

\section{Keywords}

NICRA, Climate resilient, NRM, Crop production, In-situ moisture

conservation

Article Info

Accepted:

06 May 2018

Available Online:

10 June 2018
A B S T R A C T

The NICRA project is an integrated package of proven technology which is demonstrated in Heeranar village of district Dantewada for adaptation and mitigation of the crop and livestock production systems to climate variability based on the available technologies. Demonstration of this technology is also given to the nearby villages of the adopted village. Last five years before undertaking climate resilient technologies faces climatic vulnerability such as erratic rainfalls. During 2010-2011 to 2016-17 through Village Climate Risk Management Committee (VCRMC) under the supervision of Krishi Vigyan Kendra, Dantewada some of the climate resilient technologies undertaken in farmers field. Climate resilient technologies in terms of Natural Resource Management (NRM), Crop production, Livestock and Fisheries and Institutional Interventions mainly undertaken. The result of the study shows that all the climate resilient technologies not only greatly solved the farming problems of farmers but it also boost up the economy of the farmer as well.

\section{Introduction}

Agriculture is subjected to number of stress and potential yield are seldom achieved with stress. All crops grown under natural environment are subjected to one or the other stress. Climate change can affect the yield positively as well as negatively. Agriculture, particularly in India with nearly $60 \%$ rainfed area, has been a highly risky venture with vagaries of monsoon besides the interplay of other biotic and abiotic factors. Rainfall is predicted to be highly erratic with fewer rainy days but with greater intensity. A combination of higher average annual temperatures and water stress (excess or deficit) can have serious implications for crop production in the tropics. There is positive correlation between meteorological parameters on the natural incidence of early blight disease and its severity (Dhal et al., 2016) in tomato. Cultivated varieties of eggplant are susceptible to a wide array of biotic and abiotic stress conditions.

Maximum fruit infestation $(78.45 \%)$ was recorded in 25th July, 2013 with $33.6^{\circ} \mathrm{C}$ temperature and $85 \%$ Relative Humidity; While, minimum infestation was recorded in $27^{\text {th }}$ June, 2013 i.e. $36.84 \%$ with $34.9^{\circ} \mathrm{C}$ temperature and $74 \%$ Relative Humidity (Rattan et al., 2016). The frequency of occurrence of extreme weather events such as tropical cyclones and heat waves is on the rise 
(NATCOM, 2004; IPCC, 2007) and compounds the adverse effects on agriculture. It is therefore most important that we enhance the resilience of Indian Agriculture production system to climate variability and climate change. Resilience is the capacity of the production system to resist the negative impacts of climate change and also the capacity to recover quickly after the damage.

The Indian Council of Agricultural Research (ICAR), New Delhi has launched a major network project entitled, National Initiative on Climate Resilient Agriculture (NICRA) during 2010-11, focusing on the process of developing district level contingency plans for all the rural districts of country with Central Research Institute for Dry land Agriculture (CRIDA), Hyderabad as the nodal agency.

The project is implemented by Krishi Vigyan Kendras (KVKs) at district level, regionally coordinated by the Zonal Project Directorates (ZPDs) with overall planning, monitoring and coordination by CRIDA and is being implemented at large number of Research Institutes of ICAR, State Agricultural Universities and $100 \mathrm{KVKs}$, in which KVK Dantewada is one of them (Jasna et al., 2014).

NICRA has the major objectives, to enhance the resilience of Indian agriculture covering crops, livestock and fisheries to climatic variability and climate change through development and application of improved production and risk management technologies; to demonstrate site specific technology packages on farmers' fields for adapting to current climate risks; and to enhance the capacity building of scientists and other stakeholders in climate resilient agricultural research and its application. Both short term and long terms outputs are expected from the project in terms of new NICRA has the major objectives, to enhance the resilience of Indian agriculture.

\section{Materials and Methods}

The present study is conducted at Heeranar village under Dantewada district of Chhattisgarh. The geographical location of the village falls between $18^{\circ} \mathrm{N}-19^{\prime} \mathrm{N}$ to $81^{\circ} 23^{\prime}$ $035 \mathrm{E}$ and the elevation of the village is $404 \mathrm{~m}$. The village Heeranar falls under Bastar plateau agro climatic zone of Chhattisgarh. The average rainfall of the village is 1159.3 $\mathrm{mm}$. The soils mostly in this village are Entisols and Inceptisols. The major crops grown in this village are paddy, minor millets, black gram, niger etc. Total cultivated area comes around 282.21 ha. The major climate challenges encounters are prolong dry spell like situation (drought). Erratic rainfall and early withdrawal of monsoon are the major constraint of the adopted village and due to that drought like situation occurs. There are three major topography of the land i.e. Upland, Midland and low land locally known as Marhan, Tikra and Gabhar respectively. Majority of area comes under upland in which most of the farmers grow mid and early duration traditional rice. Krishi Vigyan Kendra, Dantewada adopted the Heeranar village under NICRA project during 2010-11 and demonstrated the high yielding and short duration varieties of cereals, pulses, oilseed and vegetables to escape the hazardous effect of drought like situation. It also promotes the livelihood option like poultry farming, fish farming, goatery, dairy, mushroom cultivation etc. Mechanized agricultural practices i.e. use of seed-cum-ferti drill, cultivator, rotavator, reaper, hand hoe, threasure etc not only improves the productivity of the crop but it saves time and labour as well. NICRA has set up the custom hiring centre in the adopted village for promotion of agricultural implements in crop production.

Rainfall in Dantewada district mostly occurs through south east monsoon and most of the rainfall occurs from June to August. KVK 
promoted farm ponds, stop dam, check dam, ring wells, open wells like water harvesting structure to conserve the rain water under NICRA project.

The stored water provides lifesaving irrigation, recharge ground water and increase the irrigated area of the village. In-situ moisture conservation practices like use of mulching, SRI method of rice cultivation, line sowing by seed drill etc. was also promoted under this scheme. Green manuring, Nadep and vermicompost tanks were constructed to increase the soil fertility.

\section{Results and Discussion}

The following climate resilient agricultural intervention was adopted to upset the climatic extravaganzas in farmer's field during the period 2010- 2011 to 2016-2017 (Table 1-14).

Intervention modules of technology demonstration

The NICRA project is an integrated package of proven technology which is demonstrated in Heeranar village of district Dantewada for adaptation and mitigation of the crop and livestock production systems to climate variability based on the available technologies.

Demonstration of this technology is also given to other farmers from different village of Dantewada district.

Under the technology demonstration component, interventions are generally undertaken in four modules in order to address the climatic vulnerabilities. While the specific interventions for each village would be based on the needs and requirements, and climatic vulnerability of that particular village. Availability of resources also theater a role in determining the interventions. The four modules of intervention are briefed below:

\section{Module I: Natural resource management}

This module consists of interventions related to rain water harvesting i.e. renovation of stop dam and open wells, construction of RCC wells and form ponds and in-situ moisture conservation i.e. biomass mulching, green manuring etc.

\section{Renovation of stop dam and open well}

Two existing old stop dams and five open wells were renovated with the purpose of providing lifesaving irrigation and increase the cropping intensity by increase the irrigated area.

The cropping intensity of nearby field was increased up to $116 \%$, which was $105 \%$ previously and productivity of rice and green gram was increased up to $21.79 \%$ \& $24.93 \%$ respectively over the farmer's practice due to availability of water for longer period. Farmers use open wells for round the year vegetable cultivation under badi system.

\section{Farm pond and percolation tank}

Two Farm pond having size of $40 \mathrm{~m}$ X 40m X $2.5 \mathrm{~m}$ (length $\mathrm{X}$ width $\mathrm{X}$ Depth) and a percolation tank (15m X $15 \mathrm{~m} \mathrm{X} 1.8 \mathrm{~m})$ were constructed with the object to recharge ground water, RWH and provide live saving irrigation during critical stages of crop. Construction of pond increased the irrigated area up to 6.0 ha which enabled farmers to grow rice and vegetable resulting in increased yield of rice and vegetable up to $21.23 \%$ and $35.69 \%$ respectively.

\section{RCC ring well}

RCC ring wells measuring 6' diameter \& 30' deep were constructed with the purpose to recharge ground water and increase double cropped area. The irrigated area was increased 
up to 1.0 ha which provide opportunities to take vegetable during Kharif and Rabi season by increased water availability up to march.

\section{In-situ moisture conservation}

In situ soil moisture conservation in order to resilience to intermittent dry spells during crop growth period were done by using biomass mulch, plastic mulch, green manuring and seed drill etc.

\section{Module II: Crop production}

Promotion of drought tolerance and short duration varieties of paddy

In the NICRA village majority of cultivated land comes under up \& mid-land and farmers were cultivating long duration (130-140 days) traditional varieties that results in reduced productivity of the paddy. KVK demonstrated the drought tolerance variety i.e. Indira Barani dhan-1 (110-115 days), which produce sustainable yield under low rainfall condition. KVK also demonstrated some short duration varieties i.e. Sahbhagi (110-115 days), MTU 1010 (115-120 days) and Poornima (95-100 days). The rainfall data from previous five year revealed that there is lack of rainfall from September, which reduces the productivity of local long and medium duration traditional varieties. Under such situation the demonstrated variety produce sustainable yield. The results are given in the table-7.

\section{Demonstration of blast tolerance variety of finger millet}

Finger millet (Eleusine coracana (L.) Gaertn. ssp. coracana) is an important food, food security and cash crop in eastern and southern Africa where small-scale farmers grow it in low input farming systems. The crop has food security, nutritional, cultural, medicinal, and economic value with high industrial potential.
The traditional variety growing by farmers of Dantewada district are suffer from blast and reduction in yield is about $30-50 \%$ as per severity of disease. KVK demonstrated high yielding and blast tolerance variety Indira Ragi-1 under the NICRA project. In this variety the incidence of blast is negligible and having good yield potential.

\section{Promotion of short duration and high yielding varieties of pulses}

In the NICRA adopted village, the farmers were using mostly paddy in upland locally name as Marhan and some farmers were using long duration traditional black gram, which has very low yield potential. The soil of this upland is sandy having very low water holding capacity and not suitable for paddy cultivation. The NICRA village received sufficient amount of rainfall $(1162 \mathrm{~mm}$ annually) but the erratic nature of monsoon does not allow the paddy cultivation under upland situation. About $85 \%$ of rainfall occurs from June to August and very little rainfall during rest of the month. Looking to above situation KVK promoted the short duration and high yielding variety TAU-2 of black gram.

An area of 9.5 ha was converted from paddy and local black gram to short duration high yielding (TAU-2) variety of black gram in the adopted village and about 20 ha in the adjoining villages.

\section{Demonstration of triple resistance hybrid Arka Rakshak of tomato}

Bacterial wilt and leaf curl in tomato is very serious problem in the district and adopted village as well. Yield reduction up to $75 \%$ was observed due to this disease. Looking to the problem Arka Rakshak hybrid tomato was demonstrated in the adopted village and adjoining villages. 
Table.1 Impact of stop dam on crop productivity

\begin{tabular}{|c|c|c|c|c|c|c|c|c|c|}
\hline \multirow{2}{*}{$\begin{array}{l}\text { Irrigated } \\
\text { area } \\
\text { increased }\end{array}$} & \multirow{2}{*}{$\begin{array}{l}\text { Growing } \\
\text { crops }\end{array}$} & \multirow{2}{*}{$\begin{array}{c}\text { Availability } \\
\text { of water }\end{array}$} & \multirow{2}{*}{$\begin{array}{l}\text { No. of } \\
\text { Benefici } \\
\text { aries }\end{array}$} & \multirow{2}{*}{$\begin{array}{l}\text { Major } \\
\text { crop }\end{array}$} & \multirow[t]{2}{*}{ Variety } & \multirow{2}{*}{$\begin{array}{l}\text { Area } \\
\text { (ha.) }\end{array}$} & \multicolumn{2}{|c|}{ Yield (q/ha.) } & \multirow{2}{*}{$\begin{array}{c}\% \\
\text { increase } \\
\text { in yield }\end{array}$} \\
\hline & & & & & & & FP & $\mathrm{RP}$ & \\
\hline \multirow{2}{*}{$\begin{array}{c}\text { up to } 8.0 \\
\text { ha }\end{array}$} & \multirow{2}{*}{$\begin{array}{l}\text { Rice, Green } \\
\text { Gram, } \\
\text { Vegtables etc. }\end{array}$} & \multirow{2}{*}{$\begin{array}{c}\text { Up to end of } \\
\text { April }\end{array}$} & \multirow[t]{2}{*}{22} & Rice & Samleshwari & 4.5 & 24.5 & 29.84 & 21.79 \\
\hline & & & & $\begin{array}{l}\text { Green } \\
\text { gram }\end{array}$ & Pusa Vishal & 2.0 & 7.3 & 9.12 & 24.93 \\
\hline
\end{tabular}

Table.2 Impact of open wells on crop productivity

\begin{tabular}{|c|c|c|c|c|c|c|c|c|c|}
\hline $\begin{array}{l}\text { Irrigated } \\
\text { area } \\
\text { increased }\end{array}$ & $\begin{array}{c}\text { Availab } \\
\text { ility of } \\
\text { water }\end{array}$ & $\begin{array}{l}\text { No. of } \\
\text { Benefi } \\
\text { ciaries }\end{array}$ & $\begin{array}{l}\text { Growing } \\
\text { crops }\end{array}$ & $\begin{array}{l}\text { Major } \\
\text { crop }\end{array}$ & Variety & $\begin{array}{c}\text { Rainfa } \\
\text { Il } \\
(\mathrm{mm})\end{array}$ & $\begin{array}{l}\text { Water used } \\
\text { for critical } \\
\text { irrigation } \\
\quad(\mathrm{cm})\end{array}$ & $\begin{array}{c}\text { Yield } \\
\text { (q/ha.) }\end{array}$ & $\begin{array}{c}\text { WUE } \\
\text { (Kg/ha- } \\
\text { mm) }\end{array}$ \\
\hline \multirow{2}{*}{$\begin{array}{c}\text { Up to } 2.0 \\
\text { ha. during } \\
\text { kharif \& } \\
\text { Rabi. }\end{array}$} & \multirow{2}{*}{$\begin{array}{l}\text { Up to } \\
\text { march. }\end{array}$} & \multirow[t]{2}{*}{05} & \multirow[t]{2}{*}{ vegetables } & Tomato & Hy.Laxmi & 7.49 & 46.29 & 302.00 & 64.20 \\
\hline & & & & Brinjal & Muktakeshi & 7.49 & 48.42 & 289.30 & 58.83 \\
\hline
\end{tabular}

Table.3 Economical impact of farm pond

\begin{tabular}{|c|c|c|c|c|c|c|c|c|c|}
\hline \multirow{2}{*}{$\begin{array}{l}\text { Irrigated } \\
\text { area } \\
\text { increased }\end{array}$} & \multirow{2}{*}{$\begin{array}{c}\text { Availability } \\
\text { of water }\end{array}$} & \multirow{2}{*}{$\begin{array}{c}\text { No. of } \\
\text { Beneficiaries }\end{array}$} & \multirow{2}{*}{$\begin{array}{l}\text { Growing } \\
\text { crops }\end{array}$} & \multirow{2}{*}{$\begin{array}{l}\text { Major } \\
\text { crop }\end{array}$} & \multirow[t]{2}{*}{ Variety } & \multirow{2}{*}{$\begin{array}{l}\text { Area } \\
\text { (ha.) }\end{array}$} & \multicolumn{2}{|c|}{ Yield (q/ha.) } & \multirow{2}{*}{$\begin{array}{c}\% \\
\text { increase } \\
\text { in yield }\end{array}$} \\
\hline & & & & & & & FP & $\mathbf{R P}$ & \\
\hline \multirow{2}{*}{$\begin{array}{c}\text { Up to } 6.0 \\
\text { ha. During } \\
\text { kharif \& } 2.5 \\
\text { ha. During } \\
\text { Rabi }\end{array}$} & \multirow{2}{*}{$\begin{array}{c}\text { Up to end of } \\
\text { March }\end{array}$} & \multirow[t]{2}{*}{20} & \multirow{2}{*}{$\begin{array}{c}\text { Paddy \& } \\
\text { vegetables }\end{array}$} & Rice & MTU -1010 & 3.5 & 29.20 & 35.40 & $21 . .23$ \\
\hline & & & & Tomato & Hy. Laxmi & 1.0 & 45.46 & 161.2 & 254.59 \\
\hline
\end{tabular}

Table.4 Impact of RCC ring wells

\begin{tabular}{|c|c|c|c|c|c|c|c|c|c|}
\hline $\begin{array}{l}\text { Irrigated } \\
\text { area } \\
\text { increased }\end{array}$ & $\begin{array}{c}\text { Availab } \\
\text { ility of } \\
\text { water }\end{array}$ & $\begin{array}{l}\text { No. of } \\
\text { Benefi } \\
\text { ciaries }\end{array}$ & $\begin{array}{l}\text { Growing } \\
\text { crops }\end{array}$ & $\begin{array}{c}\text { Major } \\
\text { crop }\end{array}$ & Variety & $\begin{array}{c}\text { Rainfa } \\
\text { ll } \\
\text { (mm) }\end{array}$ & $\begin{array}{l}\text { Water used } \\
\text { for critical } \\
\text { irrigation } \\
\quad(\mathrm{cm})\end{array}$ & $\begin{array}{c}\text { Yield } \\
\text { (q/ha.) }\end{array}$ & $\begin{array}{l}\text { WUE } \\
\text { (Kg/ha- } \\
\text { mm) }\end{array}$ \\
\hline \multirow{2}{*}{$\begin{array}{l}\text { Up to } 1.0 \\
\text { ha. during } \\
\text { kharif \& } \\
\text { Rabi. }\end{array}$} & \multirow{2}{*}{$\begin{array}{l}\text { Up to } \\
\text { march. }\end{array}$} & \multirow[t]{2}{*}{05} & \multirow[t]{2}{*}{ vegetables } & Tomato & Hy.Laxmi & 7.49 & 40.96 & 405.65 & 99.03 \\
\hline & & & & Brinjal & $\begin{array}{l}\text { Muktakes } \\
\text { hi }\end{array}$ & 7.49 & 42.74 & 280.50 & 65.62 \\
\hline
\end{tabular}

Table.5 Impact of in-situ moisture conservation practices

\begin{tabular}{|l|c|c|c|c|}
\hline $\begin{array}{l}\text { In-situ moisture } \\
\text { practices }\end{array}$ & $\begin{array}{c}\text { No. of } \\
\text { demonstration }\end{array}$ & $\begin{array}{c}\text { Area } \\
\text { (ha.) }\end{array}$ & $\begin{array}{l}\text { Yield } \\
\text { (q/ha) }\end{array}$ & $\begin{array}{c}\text { WUE } \\
\text { (Kg/ha-mm) }\end{array}$ \\
\hline Green manuring and SRI & 49 & 19.6 & 46.21 & 4.20 \\
\hline DSR & 105 & 42.0 & 29.54 & 3.29 \\
\hline $\begin{array}{l}\text { Drip irrigation with plastic mulch of } \\
\text { Tomato }\end{array}$ & 19 & 7.6 & 695.68 & 192.22 \\
\hline
\end{tabular}


Table.6 Impact of SRI

\begin{tabular}{|l|c|c|c|c|c|c|}
\hline \multicolumn{1}{|c|}{ System } & $\begin{array}{c}\text { Plant } \\
\text { height } \\
(\mathbf{c m})\end{array}$ & $\begin{array}{c}\text { Number of } \\
\text { tillers/hills }\end{array}$ & $\begin{array}{c}\text { No. of grains } \\
\text { per panicle }\end{array}$ & $\begin{array}{c}\text { Yield } \\
(\mathbf{q} / \mathbf{h a})\end{array}$ & $\begin{array}{c}\text { WUE } \\
\text { (Kg/ha- } \\
\text { mmm) }\end{array}$ & B:C Ratio \\
\hline $\begin{array}{l}\text { SRI var. MTU } \\
\text { 1010 }\end{array}$ & 88.56 & 17.56 & 88.47 & 42.52 & & 3.35 \\
\hline $\begin{array}{l}\text { Conventional } \\
\text { (Broadcasting) }\end{array}$ & 83.49 & 6.95 & 71.68 & 22.69 & & 2.26 \\
\hline \% increased & 6.07 & 152.66 & 23.42 & 87.39 & & 48.23 \\
\hline
\end{tabular}

Table.7 Impact of drought tolerance and short duration varieties of rice

\begin{tabular}{|l|c|c|c|c|c|c|}
\hline Paddy variety & $\begin{array}{c}\text { Plant } \\
\text { height } \\
\text { (cm) }\end{array}$ & $\begin{array}{c}\text { Number of } \\
\text { effective } \\
\text { tillers/hills }\end{array}$ & $\begin{array}{c}\text { No. of grains } \\
\text { per panicle }\end{array}$ & $\begin{array}{c}\text { Duration } \\
\text { (Days) }\end{array}$ & $\begin{array}{c}\text { Yield } \\
\text { (q/ha) }\end{array}$ & B:C Ratio \\
\hline $\begin{array}{l}\text { Indira Barani } \\
\text { dhan-1 }\end{array}$ & 86.39 & 9.86 & 85.23 & $110-115$ & 34.65 & 2.66 \\
\hline MTU-1010 & 85.69 & 9.91 & 91.68 & $115-120$ & 35.51 & 2.80 \\
\hline Sahbahgi & 90.26 & 7.02 & 75.26 & $110-115$ & 29.06 & 2.29 \\
\hline Poornima & 85.16 & 5.62 & 66.35 & $95-100$ & 27.46 & 2.16 \\
\hline $\begin{array}{l}\text { Traditional } \\
\text { paddy }\end{array}$ & 98.69 & 3.16 & 45.62 & $135-140$ & 21.11 & 1.66 \\
\hline
\end{tabular}

Table.8 Impact of blast tolerance variety of finger millet

\begin{tabular}{|l|c|c|c|c|c|}
\hline Name of Variety & $\begin{array}{c}\text { Duration } \\
\text { (Days) }\end{array}$ & $\begin{array}{c}\text { Blast severity } \\
(\%)\end{array}$ & $\begin{array}{c}\text { Number of } \\
\text { tillers/hills }\end{array}$ & Yield (q/ha) & B:C Ratio \\
\hline Indira Ragi-1 & $130-135$ & 2.16 & 7.59 & 12.56 & 2.61 \\
\hline Traditional Ragi & $120-140$ & 34.23 & 3.12 & 6.26 & 1.30 \\
\hline \% increased & - & 299.1 & 143.26 & 100.63 & 100.76 \\
\hline
\end{tabular}

Table.9 Economical impact of black gram

\begin{tabular}{|l|c|c|c|c|c|}
\hline $\begin{array}{l}\text { Name } \\
\text { Variety }\end{array}$ & $\begin{array}{c}\text { Duration } \\
\text { (Days) }\end{array}$ & $\begin{array}{c}\text { No. of branches /plant at } \\
\text { 60 DAS }\end{array}$ & $\begin{array}{c}\text { Number of } \\
\text { pods/plant }\end{array}$ & $\begin{array}{c}\text { Yield } \\
\text { (q/ha) }\end{array}$ & B:C Ratio \\
\hline TAU-2 & $70-75$ & 4.98 & 45.22 & 8.21 & 3.07 \\
\hline $\begin{array}{l}\text { Traditional } \\
\text { black gram }\end{array}$ & $85-90$ & 2.81 & 29.87 & 4.96 & 1.98 \\
\hline \% increased & - & 77.22 & 51.38 & 65.52 & 55.05 \\
\hline
\end{tabular}

Table.10 Impact of triple resistance hybrid Arka Rakshak of tomato

\begin{tabular}{|l|c|c|c|c|}
\hline Tomato cultivars & Disease intensity (\%) & Fruits/plant (No.) & Yield (q/ha) & B:C Ratio \\
\hline Arka Rakshak & 0.00 & 47.99 & 354.64 & 3.47 \\
\hline $\begin{array}{l}\text { Farmers practice } \\
\text { (Hy. Laxmi) }\end{array}$ & 17.0 & 29.79 & 212.13 & 2.07 \\
\hline $\begin{array}{l}\text { \% change over } \\
\text { control }\end{array}$ & - & 61.09 & 95.79 & 67.63 \\
\hline
\end{tabular}


Table.11 Economics of fish farming in community pond

\begin{tabular}{|c|c|c|c|c|c|}
\hline Fish species & Yield (kg/ha) & $\begin{array}{c}\text { Gross } \\
\text { Income }\end{array}$ & $\begin{array}{c}\text { Cost of } \\
\text { Cultivation }\end{array}$ & Net Income & B:C ratio \\
\hline Rohu, Katla and Mrigal & 178 & 28480.00 & 4650.00 & 23830.00 & 6.12 \\
\hline
\end{tabular}

Table.12 Economics of Kadaknath poultry and broiler farming under backyard condition

\begin{tabular}{|c|c|c|}
\hline Particulars & $\begin{array}{l}\text { Kadaknath poultry } \\
\text { (Unit } 50 \text { birds) }\end{array}$ & $\begin{array}{l}\text { Broiler poultry } \\
\text { (Unit } 50 \text { birds) }\end{array}$ \\
\hline \multicolumn{3}{|l|}{ A. Cost (Rs.) } \\
\hline$>\quad$ Cost of Chicks for 50 chicks & $\begin{array}{c}2500=00 \\
\text { (Rs. 50/ bird) }\end{array}$ & $\begin{array}{c}1250=00 \\
\text { (Rs. } 25 / \text { bird) }\end{array}$ \\
\hline Cost of feed for 50birds in 1 cycle & 0 & 0 \\
\hline Cost of equipments (Feeder, drinker etc.) & $200=00$ & $500=00$ \\
\hline Cost of medicine and vaccines & 0 & $400=00$ \\
\hline Housing cost & 0 & 0 \\
\hline Labour cost & 0 & 0 \\
\hline Marketing cost & 0 & 0 \\
\hline Transporting cost & 0 & 0 \\
\hline$>\quad$ Miscellaneous cost i.e. electricity etc. & 0 & 0 \\
\hline B. Total Cost (Rs.) & 2700 & 2150 \\
\hline \multicolumn{3}{|l|}{ C. Benefits (Rs.) } \\
\hline$>\quad$ Sale of birds of $1.5 \mathrm{~kg}$ weight & 45000 & 6000 \\
\hline D. Net benefits (Rs.) (D=C-B) & 42300 & 3850 \\
\hline B:C Ratio & 15.67 & 1.79 \\
\hline
\end{tabular}

Table.13 Evaluation of custom hiring of farm implements and machinery

\begin{tabular}{|c|c|c|c|c|c|c|c|}
\hline Implements & $\begin{array}{c}\text { No. of } \\
\text { Units }\end{array}$ & $\begin{array}{c}\text { Usage } \\
\text { per } \\
\text { unit } \\
\text { (hr) }\end{array}$ & $\begin{array}{c}\text { Area } \\
\text { Covered } \\
\text { (ha) }\end{array}$ & $\begin{array}{c}\text { No. of } \\
\text { Farmers } \\
\text { using } \\
\text { implement }\end{array}$ & $\begin{array}{c}\text { Labour } \\
\text { saved }\end{array}$ & $\begin{array}{c}\text { Cost } \\
\text { Saving } \\
\text { (hr/ha) }\end{array}$ & $\begin{array}{c}\text { Revenue } \\
\text { generated from } \\
\text { CHCs (Rs.) }\end{array}$ \\
\hline $\begin{array}{c}\text { Tractor drawn } \\
\text { Cultivator }\end{array}$ & 1 & 194 & 53 & 79 & 25 & 5000.00 & 13700.00 \\
\hline $\begin{array}{c}\text { Tractor drawn } \\
\text { Rotavator }\end{array}$ & 1 & 98 & 29.5 & 37 & 13 & 2600.00 & 10900.00 \\
\hline $\begin{array}{c}\text { Seed cum } \\
\text { fertilizer drill }\end{array}$ & 1 & 27 & 10 & 18 & 10 & 800.00 & 6350.00 \\
\hline $\begin{array}{c}\text { Ambika paddy } \\
\text { weeder }\end{array}$ & 2 & 240 & 12 & 30 & 31 & 6200.00 & 1750.00 \\
\hline $\begin{array}{c}\text { Thresher } \\
\text { Tractor drawn }\end{array}$ & 1 & 9 & 45 & 29 & 45 & 400.00 & 18700.00 \\
\hline Leveler & 1 & 80 & 20 & 12 & 10 & 2000.00 & 1000.00 \\
\hline $\begin{array}{c}\text { Paddy } \\
\text { transplanter }\end{array}$ & 1 & 11 & 4.6 & 8 & 20 & 400.00 & 4200.00 \\
\hline Reaper & 1 & 23 & 22 & 11 & 45 & 600.00 & 12300.00 \\
\hline Total & $\mathbf{9}$ & $\mathbf{6 8 2}$ & $\mathbf{1 9 6 . 1}$ & $\mathbf{2 2 4}$ & $\mathbf{1 9 9}$ & $\mathbf{1 8 0 0 0}$ & $\mathbf{6 8 9 0 0 . 0 0}$ \\
\hline
\end{tabular}


Table.14 Details of training programme, kisan gosthi, field day, diagnostic

\begin{tabular}{|c|c|c|c|c|c|c|c|c|}
\hline Year & $\begin{array}{c}\text { No. of } \\
\text { Training }\end{array}$ & $\begin{array}{c}\text { No. of } \\
\text { Part. }\end{array}$ & $\begin{array}{c}\text { No. of Kisan } \\
\text { Gosthi }\end{array}$ & $\begin{array}{c}\text { No. of } \\
\text { Part. }\end{array}$ & $\begin{array}{c}\text { Awareness } \\
\text { camp }\end{array}$ & $\begin{array}{c}\text { No. of } \\
\text { Part. }\end{array}$ & $\begin{array}{c}\text { Exposure } \\
\text { Visit }\end{array}$ & $\begin{array}{c}\text { No. of } \\
\text { Part. }\end{array}$ \\
\hline $\mathbf{2 0 1 0 - 1 1}$ & 07 & 235 & 3 & 44 & 1 & 34 & 1 & 45 \\
\hline $\mathbf{2 0 1 1 - 1 2}$ & 09 & 302 & 5 & 65 & 1 & 47 & 2 & 60 \\
\hline $\mathbf{2 0 1 2 - 1 3}$ & 14 & 387 & 4 & 67 & 2 & 39 & 2 & 80 \\
\hline $\mathbf{2 0 1 3 - 1 4}$ & 13 & 309 & 5 & 71 & 4 & 203 & 2 & 91 \\
\hline $\mathbf{2 0 1 4 - 1 5}$ & 16 & 410 & 7 & 89 & 4 & 251 & 1 & 53 \\
\hline $\mathbf{2 0 1 5 - 1 6}$ & 10 & 281 & 8 & 126 & 3 & 196 & 1 & 45 \\
\hline $\mathbf{2 0 1 6 - 1 7}$ & 12 & 359 & 10 & 178 & 4 & 536 & 1 & 50 \\
\hline Total & $\mathbf{8 1}$ & $\mathbf{2 2 8 3}$ & $\mathbf{4 2}$ & $\mathbf{6 4 0}$ & $\mathbf{1 9}$ & $\mathbf{1 3 0 6}$ & $\mathbf{1 0}$ & $\mathbf{4 2 4}$ \\
\hline
\end{tabular}

\section{Cropping Systems followed at NICRA Village}

\begin{tabular}{|c|c|c|c|}
\hline $\begin{array}{l}\text { Topographical } \\
\text { situation }\end{array}$ & $\begin{array}{c}\text { Area } \\
(\%)\end{array}$ & Soil Type & Cropping system \\
\hline Marhan & 30 & Entisols & Minor millets / Niger-fallow/maize - Rapseed \\
\hline Tikra & 25 & Entisols & $\begin{array}{l}\text { Upland paddy / minor millets / black gram /Pigeonpea /niger - } \\
\text { fallow }\end{array}$ \\
\hline Mal & 20 & Inceptisols & Medium duration rice varieties-fallow or chickpea/Rapseed \\
\hline Gabhar & 20 & Alfisols & $\begin{array}{l}\text { Long-duration rice varieties-fallow or Chickpea/wheat/ vegetables } \\
\text { (under irrigated conditions; very small and scattered area). }\end{array}$ \\
\hline $\begin{array}{l}\text { Homestead } \\
\text { garden/ Badi }\end{array}$ & 5 & $\begin{array}{l}\text { Entisol/ } \\
\text { Inceptisol }\end{array}$ & Vegetables/Fellow \\
\hline
\end{tabular}

Cropping pattern: Rice-fallow (monocropping)

Arka Rakshak's popularity is not just due to its high-yielding quality, but also owing to its resistance to three diseases of tomato: leaf curl virus, bacterial wilt and early blight. The resistance to triple diseases would reduce the cost of cultivation by 10 to 15 per cent in terms of savings towards the cost of fungicides and pesticides (Kumar, 2014).

Module III: Adoption and impact of climate resilient technology under fisheries and livestock

Major activities of this module are use of community pond for fish production during droughts, augmentation of fodder production through improved planting material, improved shelters for reducing heat stress in livestock, management of fish ponds/tanks during water scarcity and promotion of livestock as such as a climate change adaptation strategy.

\section{Fish production in community pond}

Three Indian major carp (rohu, catla and mrigal) were distributed for rearing in community pond of NICRA village.

\section{Backyard poultry farming}

Poultry is one of the fastest growing segments of the agricultural sector. Family poultry (or the 'traditional scavenging' system), which is based almost entirely on native birds, has been by-passed by the poultry revolution, with virtually all the growth occurring in the large-scale 'confined and intensive' subsector. By contrast, traditional poultrykeeping appears to be a stagnant lowproductivity sub-sector. The meat of family Kadaknath chicks under backyard farming produced scavenging chickens is much more highly valued (by rural and urban dwellers, 
rich and poor) than that of industrially produced birds. As part of the project programme, 10 units $(50$ birds/unit $)$ of Kadaknath chicks were distributed in the village and the farmers were encouraged to rear under semi intensive care system along with the local birds (Table 12).

\section{Preventive vaccination}

Vaccination mainly done against FMD and Ranikhet. Most of the disease outbreaks occur during winter followed by rainy and summer season. In order to prevent economic losses due to various diseases in livestock and poultry, a camp was held in the NICRA village where 316 animals were given vaccination.

Module IV: Adoption and impact of climate resilient technology under institutional interventions

\section{Custom hiring centre}

The custom hiring centre is run by a committee wherein the machineries/ equipments were made available to the farmers to complete sowing and intercultural operations in time. A custom hiring centre (CHC) was set up to ensure timely availability of farm machineries. With the existence of the custom hiring centre, the farmers could easily make use of the various machineries according to their requirements during field operations. Power tiller, ridge maker, threasure, reaper, seed-cum-ferti drill, paddy transplanter, land leveler, cono weeder and sprayer contributed significantly to the revenue generation of $\mathrm{CHC}$. Farmers could save 70-75 per cent of labour cost by using of Power tiller, seed drill, threasure and paddy transplanter. Apart from this, the farmers could save 40-45 per cent of the cost of cultivation by using other implements like Cono-weeder and wheel hoe. Most significant impact of $\mathrm{CHC}$ is also observed with the paddy threasure. Generally the farmers of Dantewada district using bullock drawn card for threshing of paddy, which requires more time and labour; Therefore, the paddy threasure were demonstrated in 2012 under NICRA and about 45 ha paddy area covered by threasure. The amount realized as rent from machines and implements are deposited in the account of VCRMC (Table 13).

\section{Training programme, Kisan Gosthi, field} day, diagnostic visit under NICRA

Capacity building and skill development were created amongst the NICRA farmers on use of drought tolerance and short duration varieties, natural resource management, in-situ moisture conservation, poultry farming, duckery, goatery, dairy farming, organic farming, plant protection etc. through training programme, Kisan gosthi, field day, diagnostic visit etc. Awareness on care of animal, vaccination, artificial insemination and soil health management were created through animal health camp and soil health camp. Year wise details of capacity building and skill development programme are given in table 14.

\section{References}

Dhal, A., Beura, S. K., Sethi, D. and Dash, S. R. 2016. Study on weather parameters in relation to Perpetuation of alternaria leaf blight disease in Tomato (Lycopersicon esculentum L.) The Bioscan 11(4): 2855-2858

IPCC. 2007. Climate Change 2007 - Impacts, Adaptation and Vulnerability Contribution of Working Group II to the Fourth Assessment Report (978 0521 88010-7 Hardback; 9780521 70597-4 Paperback)

Jasna, V. K., Sukanya Som, R. Roy Burman, R. N. Padaria and J. P. Sharma. 2014. 
Socio Economic Impact of Climate Resilient Technologies. International Journal of Agriculture and Food Science Technology 5 (3): 185-190

Kumar, B. S. Satish. 2014. Bangalore's famed tomato 'Arka Rakshak' to go global. The Hindu, Sep, 2014.

NATCOM, 2004. India's Initial National Communication to the UNFCC.
Ministry of Environment and Forests, Government of India.

Rattan, P., Kumar, S. and Salgotra, R. K. 2016. Role of abiotic factors in the incidence of fruit and shoot borer (Leucinodes orbonalis guenee) in Eggplant (Solanum melongena L.). The Bioscan 11(4): 2721-2726.

\section{How to cite this article:}

Kamal Narayan, Narayan Sahu, Premlal Sahu and Kedar Nath Yadaw. 2018. Impact of Climate Resilient Technologies on Socio-Economic Status of Farmers. Int.J.Curr.Microbiol.App.Sci. 7(06): 1269-1278. doi: https://doi.org/10.20546/ijcmas.2018.706.149 